\title{
Quantum Hamiltonian Reduction in Superspace Formalism
}

\author{
Jens Ole Madsen円 and Eric Ragoucy|́ \\ Laboratoire de Physique Théorique ENSLAPP, \\ groupe d'Annecy, LAPP, Chemin de Bellevue, B.P. 110, \\ F-74941 Annecy-le-vieux Cedex, France.
}

\begin{abstract}
Recently the quantum hamiltonian reduction was done in the case of general $s \ell(2)$ embeddings into Lie algebras and superalgebras. In this paper we extend the results to the quantum hamiltonian reduction of $N=1$ affine Lie superalgebras in the superspace formalism. We show that if we choose a gauge for the supersymmetry, and consider only certain equivalence classes of fields, then our quantum hamiltonian reduction reduces to quantum hamiltonian reduction of non-supersymmetric Lie superalgebras. We construct explicitly the super energy-momentum tensor, as well as all generators of spin 1 (and $\frac{1}{2}$ ); thus we construct explicitly all generators in the superconformal, quasi-superconformal and $\mathbb{Z}_{2} \times \mathbb{Z}_{2}$ superconformal algebras.

ENSLAPP-A-459/94

February 1994

\footnotetext{
${ }^{1}$ email : jomadsen@nbivax.nbi.dk

Address after March 1, 1994 : Niels Bohr Inst., Blegdamsvej 17, DK-2100 Copenhagen Ø, Denmark.

${ }^{2}$ email : ragoucy@lapvax.in2p3.fr
} 


\section{Introduction.}

It is well known, that for every $s \ell(2)$ embedding into a Lie (super)algebra, one can find a $W$-algebra by hamiltonian reduction of the corresponding WZNW model, see e.g. [1, 2, 3, , :1. The quantization of the $W$-algebras obtained from principal $s \ell(2)$ embeddings has been studied in a number of papers, e.g. [5, 6, 7], and for arbitrary embeddings of $s \ell(2)$ into Lie algebras the quantization of these $W$ algebras has recently been performed by de Boer and Tjin [8], using the BRST formalism as well as the theory of spectral sequences. For general $s \ell(2)$ embeddings into Lie superalgebras the problem is slightly more complicated, because of the necessity in many cases to introduce auxiliary fields, but this problem was solved in [9, 10].

In [11, 12] it was shown that if we wish to consider supersymmetric $W$ algebras in the $N=1$ superspace formalism, then we must consider instead $o s p(1 \mid 2)$ embeddings into Lie superalgebras. The purpose of this paper is to apply the methods used in [8] to the supersymmetric case, thereby quantizing the $N=1 \mathrm{~W}$ algebras, giving at the same time the so-called quantum Miura transformation that produces free-field realizations of these algebras. A description of the $N=1$ superspace formalism that we use can be found in e.g [13].

In section 2 we show the supersymmetric quantum hamiltonian reduction, using a super BRST operator. In section 3 we show that if we choose a gauge for the supersymmetry, and consider not all the fields in the operator product algebra but only certain equivalence classes of fields, then the super BRST operator reduces to the BRST operator found in [9, 10] in the case of the quantum hamiltonian reduction of non-supersymmetric Lie superalgebras. In section t we construct explicitly the super energy momentum tensor, as well as all the spin 1 generators of any $W$-algebra, and thus we construct all the generators in the superconformal, quasi-superconformal and $\mathbb{Z}_{2} \times \mathbb{Z}_{2}$ superconformal algebras [14, 15, 12], and we comment on the quantum Miura transformation. In section 5 we consider in more detail the superconformal algebra obtained from the hamiltonian reduction of $B(n \mid 1)=o s p(2 n+1 \mid 2)$, and in section 6 we sum up our results.

\section{Supersymmetric BRST Cohomology.}

In the case that we are considering, we have a basic classical simple Lie superalgebra $\mathbf{g}=\mathbf{g}_{0} \oplus \mathbf{g}_{1}, \mathbf{g}_{0}$ is the bosonic subalgebra and $\mathbf{g}_{1}$ is the fermionic subspace. We denote the generators of this Lie superalgebra by $t^{a}$. The supersymmetric, invariant bilinear form on $\mathrm{g}$ we write as $g^{a b}=\left\langle t^{a}, t^{b}\right\rangle$, and the structure constants are $\left[t^{a}, t^{b}\right]=F^{a b}{ }_{c} t^{c}([\cdot, \cdot]$ is the graded commutator of the Lie superalgebra); here and in the following summation over repeated indices is implied. For $t^{a} \in \mathbf{g}_{\alpha}, \alpha \in \mathbb{Z}_{2}$ we define $d_{a}=\alpha+1$.

We define the associated affine Lie algebra $\mathbf{g}^{(1)}$ with level $k$ in terms of the operator 
product expansions:

$$
J^{a}(Z) J^{b}(W)=\frac{k g^{a b}}{Z-W}+\frac{(\theta-\eta)}{Z-W} f_{c}^{a b} J^{c}(W)+\cdots
$$

where the dots stands for the regular part. $f^{a b}{ }_{c}$ are defined by $f^{a b}{ }_{c}=(-)^{\left(d_{a}+1\right) d_{b}} F^{a b}{ }_{c}$ [11], and the superspace variables are $Z=(z, \theta), W=(w, \eta)$. Note that the $d_{a}$ 's defined above are the usual $\mathbb{Z}_{2}$ gradings for the currents $J^{a}: J^{a}(Z) J^{b}(W)=$ $(-)^{d_{a} d_{b}} J^{b}(W) J^{a}(Z)$.

We use the tensor $g^{a b}$ and its inverse $g_{a b}$ to raise and lower indices, e.g. $f^{a b c}=$ $f^{a b}{ }_{c^{\prime}} g^{c^{\prime} c}$. We notice that we have $f^{a b c} \neq 0 \Rightarrow d_{a}+d_{b}+d_{c}=1(\bmod 2)$ and $g^{a b} \neq 0 \Rightarrow$ $d_{a}=d_{b}$.

One can show the following symmetry properties of the structure constants and the bilinear form:

$$
\begin{aligned}
f^{a b}{ }_{c} & =(-)^{d_{a} d_{b}} f^{b a}{ }_{c} \\
g^{a b} & =(-)^{d_{a}+1} g^{b a} \\
f^{a b c}=f^{a b}{ }_{e} g^{e c} & =(-)^{d_{b} d_{c}} f^{a c b} .
\end{aligned}
$$

Now let us consider an embedding of $\operatorname{ssp}(1 \mid 2)$ into the Lie superalgebra $\mathbf{g}$, and let us denote the generators corresponding to this embedding by $t^{i}, i \in\{--,-, 0,+,++\}$. Under this embedding $\mathbf{g}$ splits up into representations $\mathcal{R}_{j}$ of $\operatorname{osp}(1 \mid 2), j$ being the spin of the representation. This gives a natural grading of the generators, namely the eigenvalue under the adjoint action of $t^{0}$ (sometimes called the magnetic quantum number $m$ ). It will be convenient in most of the paper to assume that we have chosen a basis for $\mathbf{g}$ such that all the generators belong to one of the representations $\mathcal{R}_{j}$. Let us define $\Delta_{m}$ to be the set of indices of generators with grade $m: a \in \Delta_{m} \Leftrightarrow\left[t^{0}, t^{a}\right]=m t^{a}$.

We can write the current of the affine Lie superalgebra in the form $J(Z)=$ $\sum(-)^{d_{a}+1} J^{a}(Z) t_{a}$. The constraints that we wish to impose are [11, 12] $J^{-}(Z)=\chi^{-}$, $J^{a}(Z)=\chi^{a}=0$ for $a$ with negative grade, $a \neq-$, where $\chi^{-}$is some constant. For simplicity we will take $\chi^{-}=1$. We will use the convention that we write indices with negative grade with greek letters, and indices with non-negative grade with barred greek letters. We can then write the constrained current as

$$
\begin{aligned}
J(Z) & =\sum_{\alpha}(-)^{d_{\alpha}+1} \chi^{\alpha} t_{\alpha}+\sum_{\bar{\alpha}}(-)^{d_{\bar{\alpha}}+1} J^{\bar{\alpha}}(Z) t_{\bar{\alpha}} \\
& =-t_{-}+\sum_{\bar{\alpha}}(-)^{d_{\bar{\alpha}}+1} J^{\bar{\alpha}}(Z) t_{\bar{\alpha}}
\end{aligned}
$$

We now wish to quantize this constrained system. There are in principle two methods for doing this. We can impose the constraints on the classical affine Lie superalgebra, and then quantize the resulting system; or we can use the BRST formalism to impose the constraints on the quantized affine Lie superalgebra. The disadvantage of the first method is that in general the constrained system is quite complicated, and 
even though it can be quantized in some cases, there is no systematic way of doing it in the general case. On the other hand, we know very well how to quantize any affine Lie superalgebra, and there is a general method for using the BRST formalism to impose such constraints: the constrained quantum algebra is the zeroth cohomology of a certain BRST operator [16, 5].

For each constraint $\chi^{\alpha}$ we introduce a pair of superghosts $\left(B^{\alpha}, C_{\alpha}\right)$ of conformal dimensions $\left(\frac{1}{2}, 0\right)$, with Grassmann gradings $\left(d_{\alpha}+1, d_{\alpha}\right)$ and ghost numbers $(-1,1)$. We define $F(\Omega)$ to be the operator product algebra generated by the affine currents $J^{a}$ and the ghosts $B^{\alpha}$ and $C_{\alpha}$, and we note that $F(\Omega)$ is graded by the ghost number. The BRST operator that we need is then given by:

$$
\begin{aligned}
S_{B R S T} O(W) & =\oint_{W} d Z J_{B R S T}(Z) O(W), \\
J_{B R S T}(Z) & =\left(J^{\alpha}(Z)-\chi^{\alpha}\right) C_{\alpha}(Z)+\frac{1}{2}(-)^{d_{\alpha}+d_{\beta}} f_{\gamma}^{\alpha \beta}\left(B^{\gamma} C_{\beta} C_{\alpha}\right)_{0}(Z) .
\end{aligned}
$$

This is a straightforward generalization of the BRST-current given in [8]. $(\cdot)_{0}$ denotes normal ordering. Note that $J_{B R S T}$ is a bosonic current, which implies that $S_{B R S T}$ is a fermionic operator.

$S$ has ghost number one, and as mentioned above we wish to calculate the zeroth cohomology $H^{0}\left(F(\Omega), S_{B R S T}\right)$. The BRST transformations are explicitly given by the following expressions :

$$
\begin{aligned}
S_{B R S T} J^{a} & =-k g^{a \beta} D C_{\beta}+f^{a \beta}{ }_{c} J^{c} C_{\beta} \\
S_{B R S T} C_{\alpha} & =\frac{1}{2}(-)^{\left(d_{\beta}+d_{\gamma}\right)} f^{\beta \gamma}{ }_{\alpha} C_{\gamma} C_{\beta} \\
S_{B R S T} B^{\alpha} & =(-)^{d_{\alpha}}\left(J^{\alpha}+(-)^{d_{\alpha}+d_{\beta}} f^{\alpha \beta}{ }_{\gamma} B^{\gamma} C_{\beta}\right)-\chi^{\alpha}
\end{aligned}
$$

Straightforward calculations show that indeed these transformations are nilpotent.

Following [8] we now split the BRST operator into two : $S_{B R S T}=S_{0}+S_{1}$, where $J_{0}(Z)=-\chi^{\alpha} C_{\alpha}(Z)$ and $J_{1}(Z)=J^{\alpha}(Z) C_{\alpha}(Z)+\frac{1}{2}(-)^{d_{\alpha}+d_{\beta}} f_{\gamma}^{\alpha \beta}\left(B^{\gamma} C_{\beta} C_{\alpha}\right)_{0}(Z)$. If we define $\hat{J}^{a}=J^{a}+(-)^{d_{a}+d_{\beta}} f^{a \beta}{ }_{\gamma} B^{\gamma} C_{\beta}$, we can write the transformations $S_{0}$ and $S_{1}$ in the form

$$
\begin{aligned}
S_{0} \hat{J}^{a} & =-(-)^{d_{a}+d_{\beta}} f_{\gamma}^{a \beta} \chi^{\gamma} C_{\beta}=f_{-}^{a \beta} C_{\beta} \\
S_{0} C_{\alpha} & =0 \\
S_{0} B^{\alpha} & =-\chi^{\alpha}
\end{aligned}
$$

and

$$
\begin{aligned}
S_{1} \hat{J}^{a} & =-k g^{a \beta} D C_{\beta}+f^{a \beta} \hat{\gamma}^{\bar{\gamma}} C_{\beta} \\
S_{1} C_{\alpha} & =\frac{1}{2}(-)^{d_{\beta}+d_{\gamma}} f^{\beta \gamma}{ }_{\alpha} C_{\gamma} C_{\beta} \\
S_{1} B^{\alpha} & =(-)^{d_{\alpha}} \hat{J}^{\alpha} .
\end{aligned}
$$


The "hatted" generators obey the same OPE's as the unhatted ones; this is in contrast to the non-supersymmetric case, where the addition of the ghost-part changes the level. From the transformations above, we can show that $S_{0}^{2}=S_{0} S_{1}=S_{1} S_{0}=S_{1}^{2}=0$. We can now define a bi-grading of the generators of the algebra :

$$
\left.\begin{array}{rl}
\operatorname{deg}\left(J^{a}\right) & =(2 m,-2 m) \\
\operatorname{deg}\left(C_{a}\right) & =(-2 m, 2 m+1) \\
\operatorname{deg}\left(B^{a}\right) & =(2 m,-2 m-1)
\end{array}\right\} \text { for } a \in \Delta_{m} .
$$

With these definitions $D_{0}$ has grade $(1,0)$, and $D_{1}$ has grade $(0,1)$, and with this bigrading $F(\Omega)$ becomes a double complex. In the case of $s \ell(2)$ embeddings into Lie algebras, the cohomology of this complex was found in [8]. We find that the supersymmetric case is very similar, so we will only give a very brief description of the formalism. For the details we refer the reader to [8].

As we see from eq. (5) we have $S_{B R S T} B^{\alpha}=(-)^{d_{\alpha}} \hat{J}^{\alpha}-\chi^{\alpha}$ and $S_{B R S T} \hat{J}^{\alpha}=0$. In the case of Lie algebras, this implies that $F^{\alpha}(\Omega)$ generated by $\hat{J}^{\alpha}$ and $b^{\alpha}$ is a subcomplex of $F(\Omega)$. In the case of Lie superalgebras a slight complication arises, because we may have a fermionic root $\alpha$ such that $2 \alpha$ is also a root. In that case we have $\hat{J}^{\alpha}(Z) \hat{J}^{\alpha}(W)=\frac{\theta-\eta}{Z-W} f^{\alpha \alpha}{ }_{2 \alpha} \hat{J}^{2 \alpha}(W)$ and $\hat{J}^{\alpha}(Z) B^{\alpha}(W)=\frac{\theta-\eta}{Z-W} f^{\alpha{ }_{2 \alpha}} B^{2 \alpha}$. This means that $F^{\alpha}(\Omega)$ is not a subcomplex, and we must consider instead $F^{\alpha, 2 \alpha}(\Omega)$ generated by $\hat{J}^{\alpha}, \hat{J}^{2 \alpha}, B^{\alpha}$ and $B^{2 \alpha}$. One still finds, however, that the cohomology of this subcomplex is trivial: $H^{q}\left(F^{\alpha, 2 \alpha}(\Omega) ; S_{B R S T}\right)=\mathbb{C} \delta_{q, 0}$.

We now define $F_{\text {red }}(\Omega)$ to be the algebra generated by the remainder of the generators, i.e. by $\hat{J}^{\bar{\alpha}}$ and $C^{\alpha}$. One can then show that

$$
\begin{aligned}
H^{*}\left(F(\Omega) ; S_{B R S T}\right) \simeq & H^{*}\left(F_{\text {red }}(\Omega) ; S_{B R S T}\right) \otimes\left(\bigotimes H^{*}\left(F^{\alpha, 2 \alpha}(\Omega) ; S_{B R S T}\right)\right) \\
& \otimes\left(\bigotimes H^{*}\left(F^{\alpha}(\Omega) ; S_{B R S T}\right)\right) \\
= & H^{*}\left(F_{\text {red }}(\Omega) ; S_{B R S T}\right) .
\end{aligned}
$$

The next step is to find the cohomology of $F_{\text {red }}(\Omega)$. We find that $S_{0}$ annihilates $J^{\bar{\alpha}}$ only if $t^{\bar{\alpha}}$ is a highest weight in an $\operatorname{osp}(1 \mid 2)$ representation, and that $C_{\alpha}$ is $S_{0}$-trivial for all $\alpha$; thus we have

$$
H^{q}\left(F_{r e d}(\Omega) ; S_{0}\right) \simeq F_{h w}(\Omega) \delta_{q, 0}
$$

where $F_{h w}(\Omega)$ is the subspace generated by highest weight generators (generators that have regular operator product expansions with $J^{+}$). Using the theory of spectral sequences, one can show [8] that this implies

$$
H^{q}\left(F_{r e d}(\Omega) ; S_{B R S T}\right) \simeq F_{h w}(\Omega) \delta_{q, 0} .
$$

This gives us the final result for the cohomology:

$$
H^{q}\left(F(\Omega), S_{B R S T}\right) \simeq F_{h w}(\Omega) \delta_{q, 0}
$$


In order to find representatives for the elements in the cohomology, i.e. generators of the $W$ algebra, one uses the so-called tic-tac-toe construction. One starts an element of $F_{h w}(\Omega)$, a highest weight generator of grade $j: J_{h w}^{\bar{\alpha}}, \bar{\alpha} \in \Delta_{j}$. From each such generator one can construct a generator of the $W$ algebra by

$$
W^{\bar{\alpha}}(Z)=\sum_{i=0}^{2 j}(-)^{i} W_{i}^{\bar{\alpha}}(Z),
$$

where $W_{0}^{\bar{\alpha}}=J_{h w}^{\bar{\alpha}}$ and $S_{1} W_{i}^{\bar{\alpha}}=S_{0} W_{i+1}^{\bar{\alpha}}$. It is clear that the series stop as indicated at $W_{2 j}^{\bar{\alpha}}$, since this element has grade zero which implies that $S_{1} W_{2 j}^{\bar{\alpha}}=0$; in fact one can show [8] that the term $W_{2 j}^{\bar{\alpha}}$ is non vanishing for all generators $W^{\bar{\alpha}}$ in the $W$-algebra. It is easy to check that this is really an element in $H^{0}\left(F(\Omega), S_{B R S T}\right)$ and it is clear that these elements form a basis of $H^{0}\left(F(\Omega), S_{B R S T}\right)$.

\section{3 "Cohomological" Factorization of Spin $\frac{1}{2}$ Fields.}

When we consider the classical hamiltonian reduction defined by an $\operatorname{ssp}(1 \mid 2)$ embedding into a Lie superalgebra $\mathbf{g}$, one of the authors has shown [17 that if we choose a suitable gauge for the supersymmetry and factorize the spin $1 / 2$ fields in the algebra (see also [18]), the result is the $W$-algebra one gets by performing the hamiltonian reduction defined by the embedding of $s \ell(2) \subset o s p(1 \mid 2)$ into $\mathbf{g}$ in the non-supersymmetric case. A similar result can be shown in the quantum hamiltonian reduction. The comparison of the two approaches is done using a filtration of the cohomology of $S_{B R S T}$. As we will break the $N=1$ supersymmetry, we need to expand superfields into fields.

$$
\begin{array}{ll}
\widehat{J}^{a}(z, \theta)=\widehat{\psi}^{a}(z)+\theta \widehat{\jmath}^{a}(z) & J_{B R S T}(z, \theta)=j_{0}(z)+\theta j_{1}(z) \\
C_{\alpha}(z, \theta)=c_{\alpha}(z)+\theta \gamma_{\alpha}(z) & B^{\alpha}(z, \theta)=\beta^{\alpha}(z)+\theta b^{\alpha}(z)
\end{array}
$$

We also introduce $\Pi_{0}$, the subset of $\Delta_{0}$ corresponding to $\mathcal{R}_{0}$ representations:

$$
a \in \Pi_{0} \quad \Leftrightarrow \quad a \in \Delta_{0} \quad \text { and } \quad\left[t^{+}, t^{a}\right]=0
$$

and decompose the set of barred greek indices into three pieces: $\Delta_{+}, \Pi_{0}$, and $\left(\Delta_{0}-\Pi_{0}\right)$ with the notation $\underline{\alpha}, i$ and $\bar{l}$ for the three sets respectively.

At the field level, the cohomology of $S_{B R S T}$ (with current $J_{B R S T}$ ) becomes the cohomology of $s$ with current $j_{1}$. Explicitly, we have

$$
\begin{aligned}
& s\left(\widehat{\psi}^{a}\right)=-k g^{a \beta} \gamma_{\beta}+f^{a \beta} \widehat{\psi}_{\bar{\gamma}}^{\bar{\gamma}} c_{\beta}-(-)^{d_{a}+d_{\beta}} f_{\gamma}^{a \beta} \chi^{\gamma} c_{\beta} \\
& s\left(\widehat{\jmath}^{a}\right)=k g^{a \beta} \partial c_{\beta}-f^{a \beta}{ }_{\bar{\alpha}}\left(\widehat{\jmath}^{\bar{\alpha}} c_{\beta}+(-)^{d_{\bar{\alpha}}} \widehat{\psi}^{\bar{\alpha}} \gamma_{\beta}\right)+(-)^{d_{a}+d_{\beta}} f^{a \beta}{ }_{\alpha} \chi^{\alpha} \gamma_{\beta} \\
& s\left(\beta^{\alpha}\right)=(-)^{d_{\alpha}} \widehat{\psi}^{\alpha}-\chi^{\alpha} \quad s\left(b^{\alpha}\right)=(-)^{d_{\alpha}+1} \hat{\jmath}^{\alpha} \\
& s\left(\gamma_{\alpha}\right)=(-)^{d_{\beta}+d_{\mu}+1} f^{\beta \mu}{ }_{\alpha} \gamma_{\mu} c_{\beta} \quad s\left(c_{\alpha}\right)=\frac{1}{2}(-)^{d_{\beta}+d_{\gamma}} f^{\beta \gamma}{ }_{\alpha} c_{\gamma} c_{\beta}
\end{aligned}
$$


Now, we introduce a filtration of the cohomology of $s$ on $F(\Omega)=F_{0}$, i.e. a sequence of subcomplexes $F_{i}$ of $F(\Omega)$ such that $F_{0} \supset F_{1} \supset F_{2} \supset \ldots$ Here, the filtration we consider is almost trivial, since it will have only two terms $F_{0} \supset F_{1}$. We choose $F_{1}$ so that $\bar{F}=F_{0} / F_{1}$ is isomorphic to the field algebra which is the starting point in [10]. The right choice is $F_{1}$ generated by $\left\{\beta^{\alpha}, \widehat{\gamma}_{\alpha}, \widehat{\psi}^{\alpha}, \widehat{\psi}^{\underline{\alpha}}, \widehat{\psi}^{i}\right\} . \widehat{\gamma}_{\alpha}$ is constructed in such a way that $F_{1}$ is a subcomplex of $F_{0}$ :

$$
\widehat{\gamma}_{\beta}=\gamma_{\beta}-\frac{1}{k} g_{\beta \underline{\alpha}}\left(f^{\underline{\alpha} \mu}{ }_{\bar{\epsilon}} \widehat{\psi}^{\bar{\epsilon}} c_{\mu}-(-)^{d_{\underline{\alpha}}+d_{\mu}} f^{\underline{\alpha} \mu}{ }_{\epsilon} \chi^{\epsilon} c_{\mu}\right)
$$

which implies $s\left(\widehat{\psi}^{\underline{\alpha}}\right)=-k g^{\underline{\alpha} \alpha} \widehat{\gamma}_{\alpha}$. Indeed, with this definition of $F_{1}, \bar{F}$ is an affine superalgebra, generated by $\left\{\hat{\jmath}_{\perp}^{a}=\widehat{\jmath}^{a}-\frac{1}{2 k} f_{b c}^{a} \widehat{\psi}^{c} \widehat{\psi}^{b} ; b^{\alpha} ; c_{\alpha} ; \widehat{\psi}^{i}\right\}$. This superalgebra is isomorphic to the one given in [10] since $\widehat{\jmath}_{\perp}^{a}$ commute with all the $\widehat{\psi}^{a}$ and $\operatorname{dim}\left(\Delta_{0}-\right.$ $\left.\Pi_{0}\right)=\operatorname{dim} \Delta_{1 / 2}$.

On $\bar{F}$, the fields that belongs to $F_{1}$ can be set to zero. This means for example that $\gamma_{\alpha} \sim \frac{1}{k} g_{\alpha \bar{\beta}}\left(f^{\bar{\beta} \mu} \widehat{\imath}_{\bar{\imath}} \widehat{\psi}^{\bar{\imath}} c_{\mu}-(-)^{d_{\bar{\beta}}+d_{\mu}} f^{\bar{\beta}{ }_{\epsilon}} \chi^{\epsilon} c_{\mu}\right)$. Using these equivalences, one can simplify $j_{1}$, so that on $\bar{F}$,

$j_{1}(z) \sim\left(j^{\alpha}-\hat{\chi}^{\alpha}\right) c_{\alpha}-\frac{1}{2}(-)^{d_{\gamma}} f^{\alpha \beta}{ }_{\gamma} b^{\gamma} c_{\beta} c_{\alpha}-\frac{1}{k} g_{\alpha \underline{\alpha}} f^{\alpha \beta}{ }_{i} \chi^{\alpha} \widehat{\psi}^{\bar{\imath}} c_{\beta} \quad$ with $\quad \hat{\chi}^{\alpha}=\frac{\nu}{k} f_{\beta \gamma}^{\alpha} \chi^{\beta} \chi^{\gamma}$

One recognizes the form given in [10]. In particular, as $\chi^{\alpha}=\delta_{\alpha,-}, \hat{\chi}^{\alpha}$ is proportional to $\delta_{\alpha,--}$.

Thus, $H^{0}(\bar{F}, s)$ is the $W$ algebra that one gets from a quantum Hamiltonian reduction of the superalgebra $\mathbf{g}$ with respect to the $s \ell(2)$ subalgebra contained in the embedded $\operatorname{ssp}(1 \mid 2)$ algebra.

However, as $F_{1}$ is a subcomplex of $F_{0}$, we have the isomorphism: $H^{0}(\bar{F}, s) \simeq \bar{H}$ where $\bar{H}=H^{0}\left(F_{0}, s\right) / H^{0}\left(F_{1}, s\right)$. We already know that $H^{0}\left(F_{0}, s\right)$ is the supersymmetric $W$ algebra, and from

$$
\begin{aligned}
& s\left(\beta^{\alpha}\right)=\widehat{\psi}^{\alpha}-\chi^{\alpha} \Rightarrow s\left(\widehat{\psi}^{\alpha}\right)=0 \\
& s\left(\psi^{\underline{\alpha}}\right)=-k g^{\underline{\alpha} \alpha} \widehat{\gamma}_{\alpha} \Rightarrow s\left(\widehat{\gamma}_{\alpha}\right)=0 \\
& s\left(\widehat{\psi}^{i}\right)=0
\end{aligned}
$$

it is clear that $H^{0}\left(F_{1}, s\right)$ is generated by the spin $1 / 2$ fields $\widehat{\psi}^{i}$ of the supersymmetric $W$ algebrat.

Thus, $H$ is the super $W$ algebra after factorization of the spin $1 / 2$ fields. Because there is an isomorphism between $\bar{H}$ and $H^{0}(\bar{F}, s)$, we conclude that the factorized super $W$ algebra (obtained from quantum $\operatorname{osp}(1 \mid 2)$ reduction) and the $W$ superalgebra (that one gets from the quantum reduction defined by $s \ell(2) \subset o s p(1 \mid 2)$ ) are isomorphic.

\footnotetext{
${ }^{1}$ The superfields $\hat{J}^{\bar{\alpha}}$ where $\bar{\alpha} \in \Pi_{0}$ are in $H^{0}\left(F_{0}, S\right)$, see below
} 


\section{Explicit Construction of Super Generators.}

\subsection{Spin 1 Super Generators.}

It is obvious from the tic-tac-toe construction, that if the generator $t^{\bar{\alpha}} \in \Pi_{0}$, then the corresponding generator in the $W$ algebra is just $W^{\bar{\alpha}}=\hat{J}_{h w}^{\bar{\alpha}}$; in other words, the remaining super Kac-Moody part of the algebra is just the original affine current plus a ghost term: $W^{\bar{\alpha}}=J_{h w}^{\bar{\alpha}}+(-)^{d_{\bar{\alpha}}+d_{\beta}} f^{\bar{\alpha} \beta}{ }_{\gamma} B^{\gamma} C_{\beta}$.

Let us illustrate the tic-tac-toe construction by using it to find the general form of a spin 1 generator in a supersymmetric $W$-algebra. Let $\bar{\alpha}$ be an index corresponding to a highest-weight generator in a spin $\frac{1}{2}$ representation $\mathcal{R}_{\frac{1}{2}}$. We wish to find $W^{\bar{\alpha}}=$ $\sum_{i}(-)^{i} W_{i}^{\bar{\alpha}}$, where $W_{0}^{\bar{\alpha}}=\hat{J}_{h w}^{\bar{\alpha}}$ and $S_{1}\left(W_{0}^{\bar{\alpha}}\right)=S_{0}\left(W_{1}^{\bar{\alpha}}\right), S_{1}\left(W_{1}^{\bar{\alpha}}\right)=0$. Before we show the result, let us give a few technical results that are used in the calculations.

Using the supersymmetry and the invariance of the bilinear form $g^{a b}$, as well as the Jacobi identities, one can show that if $a$ and $b$ are indices for generators with osp $(1 \mid 2)$ quantum numbers $(j, m)$ and $\left(j^{\prime}, m^{\prime}\right)$ respectively, then $g^{a b} \neq 0 \Rightarrow m+m^{\prime}=0$ and $j=j^{\prime}$. For the $\operatorname{osp}(1 \mid 2)$-generators, one can even show that $g^{+a} \propto \delta_{a,-}$ and $g^{++a} \propto$ $\delta_{a,--}$. We use this property to define a nonvanishing constant $\nu$ by $g_{+a}=\nu \delta_{a,-}$. We define furthermore $N_{a}=f^{+a}{ }_{b} f^{-b_{a}}$ (no summation over $a$ ).

Another result that we will need is the relation between $(A B)_{0}(Z)$ and $(B A)_{0}(Z)$. To find this relation we need the superspace version of the Taylor expansion. We write $Z=(z, \theta), W=(w, \eta),(Z-W)=z-w-\theta \eta, D=\frac{\partial}{\partial \theta}+\theta \frac{\partial}{\partial z}$; it is not difficult to show that

$$
F(Z)=\sum_{n=0}^{\infty} \frac{1}{n !}(Z-W)^{n} \partial^{n}[F(W)+(\theta-\eta) D F(W)] .
$$

Now, given two operators $A$ and $B$, we can write the operator product expansion in the form

$$
A(Z) B(W)=\sum_{m=1}^{\infty} \frac{(A B)_{m}^{0}(W)+(\theta-\eta)(A B)_{m}^{1}(W)}{(Z-W)^{m}}+(A B)_{0}(W)+O(Z-W) .
$$

The sum terminates at $m=h_{A}+h_{B}$ (or before), where $h_{A}$ and $h_{B}$ are the conformal dimensions of the two operators. Using the superspace Taylor expansion and the fact that $A(Z) B(W)=\epsilon B(W) A(Z), \epsilon= \pm 1$, we find the following relation:

$$
(A B)_{0}=\epsilon(B A)_{0}+\epsilon \sum_{m=1}^{\infty} \frac{(-)^{m}}{m !} \partial^{m}\left\{(B A)_{m}^{0}+(\theta-\eta)\left(D(B A)_{m}^{0}-(B A)_{m}^{1}\right)\right\} .
$$

Finally we need the superspace version of the Cauchy theorem. Defining

$$
\oint_{W} d Z=\oint_{w} \frac{d z}{2 \pi i} \int d \theta,
$$

\footnotetext{
${ }^{2}$ Using the Jacobi identities one can show that if $J^{a}$ has $\operatorname{osp}(1 \mid 2)$ quantum numbers $(j, m)$, then $N_{a}=\frac{-1}{8 \nu}\left((-)^{2(j-m)}(4 j+1)-(4 m+1)\right)$.
} 
one verifies that:

$$
\begin{aligned}
& \oint_{W} d Z \frac{\theta-\eta}{(Z-W)^{(n+1)}} \Phi(Z)=\frac{1}{n !} \partial^{n} \Phi(W), \\
& \oint_{W} d Z \frac{1}{(Z-W)^{(n+1)}} \Phi(Z)=\frac{1}{n !} \partial^{n} D \Phi(W) .
\end{aligned}
$$
have

Using these results, we can now find $W^{\bar{\alpha}}=W_{0}^{\bar{\alpha}}-W_{1}^{\bar{\alpha}}, \bar{\alpha} \in \Delta_{\frac{1}{2}}$. From eq. (5) we

$$
S_{1}\left(\hat{J}^{\bar{\alpha}}\right)=-k g^{\bar{\alpha} \beta} D c_{\beta}+f^{\bar{\alpha} \beta} \hat{\gamma}^{\bar{\gamma}} c_{b}
$$

$\hat{J}^{\bar{\alpha}}$ has bigrading $(1,-1) ; S_{1}$ has bigrading $(0,1)$ and $S_{0}$ has bigrading $(1,0)$, thus terms in $W_{1}^{\bar{\alpha}}$ must have bigrading $(0,0)$. We find that

$$
\begin{aligned}
S_{0}\left(f^{-\bar{\alpha}}{ }_{\bar{\beta}} D \hat{J}^{\bar{\beta}}\right) & =\frac{1}{2} g^{\bar{\alpha} \beta} D c_{\beta}, \\
\frac{1}{\nu N_{\bar{\beta}}-m_{\bar{\beta}}} S_{0}\left(f_{\bar{\gamma} \bar{\beta}}{ }^{\bar{\alpha}} f^{-\bar{\beta}} \overline{\bar{\epsilon}}_{\bar{J}} \hat{J}^{\bar{\epsilon}} \hat{J}^{\bar{\gamma}}\right) & =-2 \nu f^{\bar{\alpha} \beta} \hat{\gamma}_{\bar{\gamma}}{ }^{\bar{\gamma}} c_{\beta} .
\end{aligned}
$$

where $\bar{\beta} \in \Delta_{m_{\bar{\beta}}}$. Note that if $t^{\bar{\beta}} \in \mathcal{R}_{j_{\bar{\beta}}}$ then $f^{-\bar{\beta}_{\bar{\epsilon}}} \neq 0 \Rightarrow j_{\bar{\beta}}, m_{\bar{\beta}}>0 \Rightarrow \nu N_{\bar{\beta}}-m_{\bar{\beta}} \neq 0$. Comparing with eq. (19) we see that

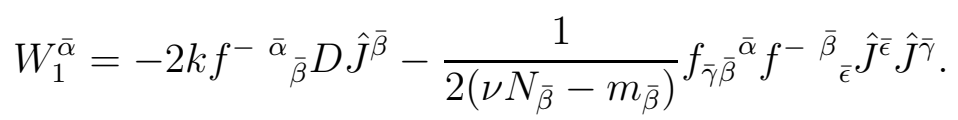

$S_{1}\left(W_{1}^{\bar{\alpha}}\right)$ vanishes, so we can now write the general form of a spin 1 generator in a supersymmetric $W$-algebra as

$$
W^{\bar{\alpha}}=\hat{J}_{h w}^{\bar{\alpha}}+2 k f^{-\bar{\alpha}}{ }_{\bar{\beta}} D \hat{J}^{\bar{\beta}}+\frac{1}{2\left(\nu N_{\bar{\beta}}-m_{\bar{\beta}}\right)} f_{\bar{\gamma} \bar{\beta}}^{\bar{\alpha}} f^{-\bar{\beta}} e_{e} \hat{J}^{e} \hat{J}^{\bar{\gamma}} . \quad \text { for } \bar{\alpha} \in \Delta_{\frac{1}{2}} .
$$

Actually the calculation for $W_{1}^{\bar{\alpha}}$ did not depend on the fact that the grade of $\bar{\alpha}$ was $1 / 2$, except for some normalizations. If $\bar{\alpha}$ is the index of a highest weight generator in a representation $\mathcal{R}_{j}, j \neq 0$, then the first equation in (20) is replaced by

$$
S_{0}\left(f^{-\bar{\alpha}}{ }_{\bar{\beta}} D \hat{J}^{\bar{\beta}}\right)=j g^{\bar{\alpha} \beta} D c_{\beta} .
$$

We see that for general $\bar{\alpha}$, where $t^{\bar{\alpha}}$ is a highest weight generator, the expression for $W^{\bar{\alpha}}$ starts with

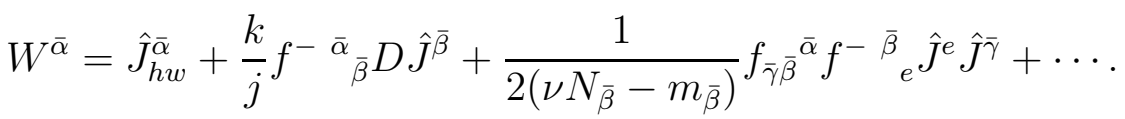

$$
\begin{aligned}
& t^{\bar{\alpha}} \in \mathcal{R}_{j}, \quad j \neq 0
\end{aligned}
$$




\subsection{Energy-Momentum Tensor and Quantum Miura Trans- formation.}

In principle we could find the complete expression for a general generator in any supersymmetric $W$ algebra obtainable by hamiltonian reduction, but the calculations quickly becomes intractable. However, in the special case of the energy-momentum tensor we can find the complete expression $W^{++}=W_{0}^{++}-W_{1}^{++}+W_{2}^{++}:\left(\mu \equiv f^{+{ }^{++}}\right.$, $\mu \neq 0)$

$$
\begin{aligned}
& W_{0}^{++}=\hat{J}^{++} \\
& W_{1}^{++}=\frac{k}{\nu \mu} D \hat{J}^{+}-\frac{1}{\nu \mu} f^{+}{ }_{\bar{\alpha} \bar{\beta}} \hat{J}^{\bar{\beta}} \hat{J}^{\bar{\alpha}} \\
& W_{2}^{++}=\frac{1}{\nu^{2} \mu}\left(2 k(k+\sigma) \partial \hat{J}^{0}-k g_{\bar{\beta}_{0} \bar{\alpha}_{0}} \hat{J}^{\bar{\alpha}_{0}} D \hat{J}^{\bar{\beta}_{0}}+\frac{1}{3} f_{\bar{\gamma}_{0} \bar{\beta}_{0} \bar{\alpha}_{0}} \hat{J}^{\bar{\alpha}_{0}} \hat{J}^{\bar{\beta}_{0}} \hat{J}^{\bar{\gamma}_{0}}\right),
\end{aligned}
$$

$\sigma$ is a contribution from the normal ordering, and can be expressed as

$$
\sigma=\nu \sum_{\bar{\alpha} \in \Delta_{0}}(-)^{d_{\bar{\alpha}}} N_{\bar{\alpha}}
$$

After normalization we find:

$$
\begin{aligned}
T= & \frac{\nu^{2} \mu}{2 k^{2}} \hat{J}^{++}-\frac{\nu}{2 k} D \hat{J}^{+}+\frac{\nu}{2 k^{2}} f_{\bar{\alpha} \bar{\beta}}^{+} \hat{J}^{\bar{\beta}} \hat{J}^{\bar{\alpha}} \\
& +\frac{k+\sigma}{k} \partial \hat{J}^{0}-\frac{1}{2 k} g_{\bar{\beta}_{0} \bar{\alpha}_{0}} \hat{J}^{\bar{\alpha}_{0}} D \hat{J}^{\bar{\beta}_{0}}+\frac{1}{6 k^{2}} f_{\bar{\gamma}_{0} \bar{\beta}_{0} \bar{\alpha}_{0}} \hat{J}^{\bar{\alpha}_{0}} \hat{J}^{\bar{\beta}_{0}} \hat{J}^{\bar{\gamma}_{0}} .
\end{aligned}
$$

As mentioned above, it is possible to show that all the generators of the $W$-algebra includes a term of zero grade. It is easy to see, that if we restrict to these zero-grade terms, we have a realization of the $W$-algebra. Thus, if we write the grade zero part of the affine Lie superalgebra in terms of free fields using the Wakimoto construction [3, we immediately get a free field realization of the $W$ algebra. This construction is called the quantum Miura transformation. In the energy momentum tensor above, the grade zero part was

$$
T_{0}=\frac{k+\sigma}{k} \partial \hat{J}^{0}-\frac{1}{2 k} g_{\bar{\beta}_{0} \bar{\alpha}_{0}} \hat{J}^{\bar{\alpha}_{0}} D \hat{J}^{\bar{\beta}_{0}}+\frac{1}{6 k^{2}} f_{\bar{\gamma}_{0} \bar{\beta}_{0} \bar{\alpha}_{0}} \hat{J}^{\bar{\alpha}_{0}} \hat{J}^{\bar{\beta}_{0}} \hat{J}^{\bar{\gamma}_{0}} .
$$

This should be compared with the classical Miura transformation of the energy momentum tensor (written in terms of the affine currents in the grade zero part) [1]:

$$
T_{\text {class }}=\partial J^{0}-\frac{1}{2 k} g_{\bar{\beta}_{0} \bar{\alpha}_{0}} J^{\bar{\alpha}_{0}} D J^{\bar{\beta}_{0}}+\frac{1}{6 k^{2}} f_{\bar{\gamma}_{0} \bar{\beta}_{0} \bar{\alpha}_{0}} J^{\bar{\alpha}_{0}} J^{\bar{\beta}_{0}} J^{\bar{\gamma}_{0}} .
$$

\footnotetext{
${ }^{3}$ In [19] the Wakimoto construction was done for Lie superalgebras in the non-supersymmetric case. To get a supersymmetric version, it suffices to add spin $1 / 2$ fermions and bosons in the adjoint representation, see 13, 20, 21. We thank K. Ito for drawing our attention to this.
} 
From equation (26) we can easily calculate the central charge of the algebra :

$$
\hat{c}=\operatorname{sdim} \mathbf{g}_{0}+\frac{2}{3 k} f^{\bar{\alpha}_{0} \bar{\beta}_{0} \bar{\gamma}_{0}} f_{\bar{\gamma}_{0} \bar{\beta}_{0} \bar{\alpha}_{0}}-8 g^{00}\left(k+2 \sigma+\frac{\sigma^{2}}{k}\right) .
$$

$\mathbf{g}_{0}$ denotes the grade zero part of the superalgebra $\mathbf{g}$, and sdim is the superdimension. We know from the normalization of the embedded $\operatorname{ssp}(1 \mid 2)$ algebra that $g^{00}= \pm \frac{1}{2}$.

Note that for those algebras which in addition to the spin $3 / 2$ super energy momentum tensor contains only a number of spin one and spin $1 / 2$ generators, the superconformal, quasi-superconformal and $\mathbb{Z}_{2} \times \mathbb{Z}_{2}$ superconformal algebras, we have found the general expressions for all generators in the algebras, and using the quantum Miura transformation it is easy to write down the corresponding free field realizations.

\section{Example: $B(n \mid 1)$.}

As an example we will take the hamiltonian reduction of $B(n \mid 1)=o s p(2 n+1 \mid 2)$. As $\operatorname{ssp}(1 \mid 2)$-subalgebra we choose the regular embedding of $\operatorname{osp}(1 \mid 2)$ into $\operatorname{ssp}(2 n+1 \mid 2)$, leading to a super Kac-Moody extended superconformal algebra [12]. We parametrize the affine current as follows:

$$
J(Z)=\left(\begin{array}{ccccccc|cc}
J^{11} & J^{12} & \cdots & J^{1, n+1} & \cdots & J^{1,2 n} & 0 & J^{1-} & J^{1+} \\
J^{21} & J^{22} & \cdots & J^{2, n+1} & \cdots & 0 & -J^{1,2 n} & J^{2-} & J^{2+} \\
\vdots & \vdots & & \vdots & & \vdots & \vdots & \vdots & \vdots \\
J^{n+1,1} & J^{n+1,2} & & 0 & & -J^{2, n+1} & -J^{1, n+1} & J^{(n+1)-} & J^{(n+1)+} \\
\vdots & \vdots & & \vdots & & \vdots & \vdots & \vdots & \vdots \\
J^{2 n, 1} & 0 & & -J^{n+1,2} & \cdots & -J^{22} & -J^{12} & J^{2 n-} & J^{2 n+} \\
0 & -J^{2 n, 1} & \cdots & -J^{n+1,1} & \cdots & -J^{21} & -J^{11} & J^{(2 n+1)-} & J^{(2 n+1)+} \\
\hline-J^{1+} & -J^{2+} & \cdots & -J^{(n+1)+} & \cdots & -J^{2 n+} & -J^{(2 n+1)+} & J^{0} & J^{++} \\
J^{1-} & J^{2-} & \cdots & J^{(n+1)-} & \cdots & J^{2 n-} & J^{(2 n+1)-} & J^{--} & -J^{0}
\end{array}\right)
$$

The fermionic roots of $\operatorname{osp}(1 \mid 2)$ are $J^{ \pm}=J^{(n+1) \pm}$. For $i+j>2 n+2$, we define $J^{i j}=-J^{2 n+2-i, 2 n+2-j}$.

The algebra splits into representations of $\operatorname{osp}(1 \mid 2)$ : a spin 1 representation $\mathcal{R}_{1}$ which is just the embedded $\operatorname{osp}(1 \mid 2)$ itself, $\mathcal{R}_{1}=\left\{J^{++}, J^{(n+1)+}, J^{0}, J^{(n+1)-}, J^{--}\right\}, 2 n$ spin $\frac{1}{2}$ representations each of the form $\mathcal{R}_{\frac{1}{2}}=\left\{J^{i+}, J^{(n+1) i}, J^{i-}\right\}, i \neq n+1$, and $n(2 n-1)$ singlet representations $\mathcal{R}_{0}=\left\{J^{i j}\right\}, i, j \neq n+1, i+j<2 n+2$.

We find that with this parametrization, the operators product expansions are $\left(Z_{12} \equiv Z_{1}-Z_{2}, \theta_{12} \equiv \theta_{1}-\theta_{2}\right.$, operators on the right hand side are taken in the $Z_{2}$ variable):

$$
J^{0}\left(Z_{1}\right) J^{0}\left(Z_{2}\right)=\frac{-k / 2}{Z_{12}}+\cdots
$$




$$
\begin{aligned}
J^{0}\left(Z_{1}\right) J^{++/--}\left(Z_{2}\right) & =\frac{\theta_{12}}{Z_{12}}\left( \pm J^{++/--}\right)+\cdots \\
J^{++}\left(Z_{1}\right) J^{--}\left(Z_{2}\right) & =\frac{-k}{Z_{12}}+\frac{\theta_{12}}{Z_{12}} 2 J^{0}+\cdots \\
J^{i j}\left(Z_{1}\right) J^{k l}\left(Z_{2}\right) & =\frac{\frac{k}{2}\left(\delta_{i l} \delta_{j k}-\delta_{j, 2 n+2-l} \delta_{i, 2 n+2-k}\right)}{Z_{12}} \\
+ & \frac{\theta_{12}}{Z_{12}} \frac{1}{2}\left(\delta_{i l} J^{k j}-\delta_{k j} J^{i l}-\delta_{i, 2 n+2-k} J^{2 n+2-l, j}+\delta_{j, 2 n+2-l} J^{i, 2 n+2-k}\right)+\cdots \\
J^{0}\left(Z_{1}\right) J^{i \pm}\left(Z_{2}\right) & =\frac{\theta_{12}}{Z_{12}}\left( \pm \frac{1}{2} J^{i \pm}\right)+\cdots \\
J^{++}\left(Z_{1}\right) J^{i-}\left(Z_{2}\right) & =\frac{\theta_{12}}{Z_{12}}\left(-J^{i+}\right)+\cdots \\
J^{--}\left(Z_{1}\right) J^{i+}\left(Z_{2}\right) & =\frac{\theta_{12}}{Z_{12}}\left(-J^{i-}\right)+\cdots \\
J^{i j}\left(Z_{1}\right) J^{k-}\left(Z_{2}\right) & =\frac{\theta_{12}}{Z_{12}} \frac{1}{2}\left(\delta_{i, 2 n+2-k} J^{(2 n+2-j)-}-\delta_{j k} J^{i-}\right)+\cdots \\
J^{i j}\left(Z_{1}\right) J^{k+}\left(Z_{2}\right) & =\frac{\theta_{12}}{Z_{12}} \frac{1}{2}\left(\delta_{i, 2 n+2-k} J^{(2 n+2-j)+}-\delta_{j k} J^{i+}\right)+\cdots \\
J^{i+}\left(Z_{1}\right) J^{j-}\left(Z_{2}\right) & =\frac{-\frac{k}{2} \delta_{i, 2 n+2-j}}{Z_{12}}+\frac{\theta_{12}}{Z_{12}} \frac{1}{2}\left(J^{2 n+2-i, j}-\delta_{i, 2 n+2-j} J^{0}\right)+\cdots \\
J^{i \pm}\left(Z_{1}\right) J^{j \pm}\left(Z_{2}\right) & =\frac{\theta_{12}}{Z_{12}}\left(\mp \frac{1}{2} \delta_{i, 2 n+2-j} J^{++/--}\right)+\cdots \\
& =30) \\
& =\cdots
\end{aligned}
$$

The constraints that we wish to impose are $J^{(n+1)-}(Z)=1$ and $J^{--}(Z)=J^{i-}(Z)=$ $0, i \neq n+1$. We introduce ghost super-fields $\left(B^{i-}, C_{i-}\right)$ and $B^{--}, C_{--} ; C_{i-}$ and $B^{--}$are bosonic while $C_{--}$and $B^{i-}$ are fermionic. The BRST currents are

$$
J_{0}(Z)=-C_{(n+1)-}(Z)
$$

and

$$
J_{1}(Z)=J^{i-}(Z) C_{i-}(Z)+J^{--}(Z) C_{--}(Z)+\frac{1}{4} B^{--} C_{i-} C_{(2 n+2-i)-}(Z) .
$$

The "hatted" currents are

$$
\begin{aligned}
\hat{J}^{++} & =J^{++} \\
\hat{J}^{i+} & =J^{i+}+B^{i-} C_{--}, \\
\hat{J}^{0} & =J^{0}+\frac{1}{2}\left(B^{i-} C_{i-}\right)_{0}-\left(B^{--} C_{--}\right)_{0}, \\
\hat{J}^{i j} & =J^{i j}+\frac{1}{2} B^{i-} C_{j-}-\frac{1}{2} B^{(2 n+2-j)-} C_{(2 n+2-i)-} \\
\hat{J}^{i-} & =J^{i-}+\frac{1}{2} B^{--} C_{(2 n+2-i)-}, \\
\hat{J}^{--} & =J^{--} .
\end{aligned}
$$


The $W$-algebra generators corresponding to the singlet representations of the $\operatorname{osp}(1 \mid 2)$ are just the hatted currents :

$$
W^{i j}=\hat{J}^{i j}=J^{i j}+\frac{1}{2} B^{i-} C_{j-}-\frac{1}{2} B^{(2 n+2-j)-} C_{(2 n+2-i)-}, i, j \neq n+1 .
$$

They form a supersymmetric so(2n)-subalgebra of the resulting $W$-algebra.

The form of the $W$-generators corresponding to the highest weights of the spin $\frac{1}{2}$ representations was given in eq. (21), and can be written as

$$
W^{i+}=\hat{J}^{i+}-k D \hat{J}^{i, n+1}-\left(\hat{J}^{i, k} \hat{J}^{k, n+1}\right)_{0}-\left(\hat{J}^{0} \hat{J}^{i, n+1}\right)_{0}
$$

Finally we have the energy momentum tensor, which after normalizations is given in eq. (25); we find

$$
\begin{aligned}
T= & -\frac{1}{k^{2}} J^{++}+\frac{1}{k} D \hat{J}^{(n+1)+}+\frac{2}{k^{2}} \hat{J}^{(n+1, j} \hat{J}^{j+}+\frac{2}{k^{2}} \hat{J}^{0} \hat{J}^{(n+1)+} \\
& +\frac{k-n+1 / 2}{k} \partial \hat{J}^{0}-\frac{1}{2 k} \operatorname{tr}(\hat{M} D \hat{M})+\frac{1}{k}\left(\hat{J}^{0} D \hat{J}^{0}\right)_{0}-\frac{1}{3 k^{2}} \operatorname{tr}(\hat{M} \hat{M} \hat{M}),
\end{aligned}
$$

where $\hat{M}$ is the "hatted" submatrix corresponding to the $s o(2 n+1)$-part in (29). The central charge is found to be $\hat{c}=4 k-8 n+5+\frac{1}{k}\left(n-\frac{1}{2}\right)^{2}$. A free field realization of the algebra can be found by inserting a free field realization of the grade zero part into the expressions above; we can take a Wakimoto realization of the super-symmetric $s o(2 n+1)^{(1)}$, and realize $J^{0}$ as a free super field $J^{0} \rightarrow \sqrt{\frac{k}{2}} D \Phi$.

\section{Conclusion.}

In this paper, we have considered the quantum hamiltonian reduction of supersymmetric affine Lie superalgebras, giving as a result the quantized supersymmetric $W$ algebras. We have quantized all the manifestly supersymmetric $W$ algebras which can be obtained by Hamiltonian reduction of affine Lie superalgebras. We have shown that using a suitable cohomological procedure, we can reduce the supersymmetric quantum hamiltonian reduction defined by an $\operatorname{osp}(1 \mid 2)$ embedding into a Lie superalgebra $\mathbf{g}$, to the non-supersymmetric hamiltonian reduction defined by the embedding of $s \ell(2) \subset \operatorname{osp}(1 \mid 2)$ into g. We have given an explicit expression for the energy momentum tensor and the central charge of all supersymmetric $W$ algebras, as well as general expressions for all generators in the superconformal, quasi-superconformal and $\mathbb{Z}_{2} \times \mathbb{Z}_{2}$ superconformal algebras. We have described the quantum Miura transformation, and described the example of the hamiltonian reduction of $\operatorname{ssp}(2 n+1 \mid 2)^{(1)}$ in some detail. 


\section{Acknowledgements}

The authors wish to acknowledge helpful discussions with F. Delduc, and to thank Kris Thielemans for supplying us with his Mathematica package for calculating super OPE's. JOM wishes to thank the ENSLAPP, where this work was done, for its kind hospitality, and the Danish Research Academy for its financial support.

\section{References}

1. M. Bershadsky and H. Ooguri. Comm. Math. Phys. 126, (1989) 49.

2. F.A. Bais, T. Tjin and P. van Driel, Nucl. Phys. BB357 (1991) 632.

3. L. Fehér, L. O'Raiffeartaigh, P. Ruelle and I. Tsutsui, Phys. Rep. 222 (1992) 1 , and references therein.

4. P. Bouwknegt and K. Schoutens, Phys. Rep. 223 (1993) 183.

5. B.L. Feigin and E. Frenkel, Phys. Lett. B246, (1990) 75.

6. P. Bouwknegt, J. McCarthy and K. Pilch, in proceedings Strings and Symmetries, N. Berkovitz et al. (eds.), World Scientific 1991.

7. E. Frenkel, V. Kac and M. Wakimoto, Comm. Math. Phys. 147 (1992) 295.

8. J. de Boer and T. Tjin, "The Relation Between Quantum W Algebras and Lie Algebras", preprint THU-93/05; Comm. Math. Phys. 158 (1993) 485.

9. A. Sevrin, K. Thielemans and W. Troost, Nucl. Phys. B407, (1993) 459.

10. A. Sevrin and W. Troost, Phys. Lett. B315, (1993) 304.

11. F. Delduc, E. Ragoucy and P. Sorba, Comm. Math. Phys. 146, (1992) 403.

12. L. Frappat, E. Ragoucy and P. Sorba, Comm. Math. Phys. 157, (1993) 499.

13. P. Di Vecchia, V.G. Knizhnik, J.L. Petersen and P. Rossi, Nucl. Phys. B253, (1985) 701.

14. E.S. Fradkin and V.Ya. Linetskii, Phys. Lett. B291 (1992) 71.

15. K. Ito, J.O. Madsen and J.L. Petersen, String Theory, Quantum Gravity and the Unification of the Fundamental Interactions, M. Bianchi, F. Fucito, E. Marinari and A. Sagnotti (eds.), World Scientific 1993, p. 302;

K. Ito, J.O. Madsen and J.L. Petersen, Phys. Lett. B318 (1993) 315.

16. B. Kostant and S. Sternberg, Ann. Phys. 176 (1987) 49.

17. E. Ragoucy, Nucl. Phys. B411, (1994) 778.

18. A. Deckmyn and K. Thielemans, "Factoring out Free Fields", preprint KULTH-93-26.

19. K. Ito, Int. J. Mod. Phys. A7, (1992) 4885.

20. J. Fuchs, Nucl. Phys. B318, (1989) 631.

21. Goddard, Olive and Waterson Comm. Math. Phys. 112, (1987) 591. 